This is the author's copy of the publication as archived with the DLR's electronic library at http://elib.dlr.de. Please consult the original publication for citation.

\title{
Analysis of task decoupling characteristics of null space projector with uncertainty from modelling error
}

Yun, Wonbum; Lee, Jinoh; Oh, Sehoon

\section{Copyright Notice}

(C)2022 IEEE. Personal use of this material is permitted. Permission from IEEE must be obtained for all other uses, in any current or future media, including reprinting/republishing this material for advertising or promotional purposes, creating new collective works, for resale or redistribution to servers or lists, or reuse of any copyrighted component of this work in other works.

\section{Citation Notice}

Qinproceedingsfyun2022analysis,

title $=\{$ Analysis of task decoupling characteristics of null space projector with uncertainty from modelling error $\}$,

author $=\{$ Yun, Wonbum and Lee, Jinoh and $0 \mathrm{~h}$, Sehoon $\}$,

booktitle $=\{2022$ IEEE/SICE International Symposium on System Integration (SII) $\}$,

pages $=\{963-964\}$

year $=\{2022\}$ 


\title{
Analysis of task decoupling characteristics of null space projector with uncertainty from modelling error
}

\author{
Wonbum Yun ${ }^{1}$, Jinoh $\mathrm{Lee}^{2}$ and Sehoon $\mathrm{Oh}^{*}$
}

\begin{abstract}
Methods for designing a multitask controller for redundant robots are often implemented by a null space projector. Although null-space projection-based controllers can achieve the task decoupling performance among prioritized tasks, it is vulnerable to uncertainty due to modeling errors, in practice. Accordingly, this paper provides analytical proof of the task coupling effect caused by the uncertainty and verifies it through a simulation.
\end{abstract}

\section{INTRODUCTION}

To design the multitask controller for the redundant robot, coupling between each tasks should be eliminated properly. To decouple the tasks, methods with the null space projector have been proposed [1] [2]. The null space projector has the effect of preventing a task coupling from the lower priority task which intervenes the task that has high priority. In this respect, the null space makes it possible to decouple tasks by setting priority to each task. The null space projection-based task decoupling controller has been applied to researches for controlling the robot which has a massive number of redundancy such as humanoids [3].

In order to decouple the tasks properly, it is important to know the exact dynamic and kinematic models of the robot which is used to design the null space projector. However, it is hard to obtain the precise mechanical properties of the robot in practice. In this sense, difference between estimated null space and the real robot's null space due to modeling error makes coupling effect between tasks. The task coupling effect caused by uncertainty should be considered for guaranteeing task decoupling performance, but it is not treated as an important issue yet.

In this paper, the performance of the task decoupling controller with uncertainty from modeling error was specified precisely. First, the characteristics of the task decoupling controller with a null space projector are organized, and the effect of uncertainty is specified. Second, the characteristics of the controller affected by the uncertainty classified as kinematic and dynamic modeling errors are analyzed analytically. Finally, the above features are shown through numerical analysis.

*This work has supported by the National Research Foundation of Korea(NRF) grant funded by the Korea government(MSIT)(NRF2019R1A2C2011444)(Corresponding author : Sehoon Oh)

${ }^{1}$ Wonbum Yun and ${ }^{*}$ Sehoon Oh are with Department of Robotics Engineering, DGIST, Daegu, Korea dnjsqjadbs@dgist.ac.kr; sehoonedgist.ac.kr

${ }^{2}$ Jinoh Lee is with the institute of Robotics and Mechatronics, German Aerospace Center (DLR), 82234 Weßling, Germany Jinoh. Leedalr.de

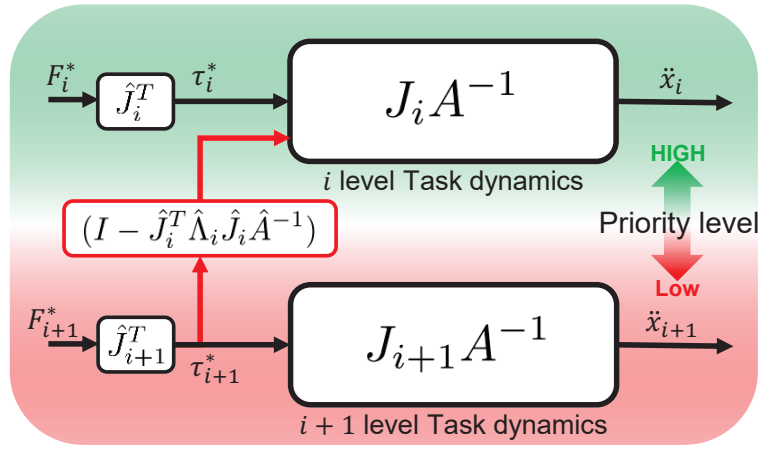

Fig. 1. Block diagram of tasks : from control forces to task responses. Red box is the null space projector that blocks task coupling effects.

\section{PRELIMINARY AND FundAMENTAL}

\section{A. Prioritized control}

Prioritized task control prevents coupling force from tasks with lower priority by using null space projection. The input torque for prioritized task control is as follows:

$$
\tau^{*}=\tau_{1}^{*}+N_{2}^{T}\left(\tau_{2}^{*}+N_{3}^{T}\left(\tau_{3}^{*}+\cdots N_{n}^{T} \tau_{n}^{*}\right)\right)
$$

The null space projector for controlling $i$ level task is

$$
\begin{array}{r}
N_{i+1}(q)=I-J_{i}^{+} J_{i} \\
J_{i}^{+}=W^{-1} J_{i}^{T}\left(J_{i} W^{-1} J_{i}^{T}\right)^{-1}
\end{array}
$$

$J_{i}^{+}$is pseudo inversion of jacobian matrix and the weighting matrix, W, satisfies $J J^{+}=I$ [2].

To yield the second-order error dynamics, the control force is designed as follows:

$$
F_{i}^{*}=\hat{\Lambda}_{i}\left(\ddot{x}_{i}^{d}+k_{d}\left(\dot{x}_{i}^{d}-\dot{x}_{i}\right)+k_{p}\left(x_{i}^{d}-x_{i}\right)\right)
$$

Control torque of $i$ level task is designed as

$$
\tau_{i}^{*}=\hat{J}_{i}^{T} F_{i}^{*}
$$

With the null space projector, the 'consistency' that the task does not be affected by other tasks can be implemented.

\section{MAIN RESUlt}

\section{A. Dynamic consistent null space projection}

A state in which the acceleration response of a certain task does not be affected by the control torque of other tasks is called 'dynamic consistency' [4]. The $i$ level task response represented in fig. 1 is defined as follows:

$$
\ddot{x}_{i}=J_{i} A^{-1}\left(\tau_{i}^{*}+\hat{N}_{i+1}^{T} \tau_{i+1}^{*}\right)
$$

The null space projector is represented as estimated value $\hat{N}$, and $A$ is the joint space inertia matrix of the robot. 
Substituting (6) with (2) and (3) and using the weighting matrix as the joint space's inertia matrix $(W=A)$, the response of $i$ level task from $i+1$ level task's torque becomes

$$
\ddot{x}_{i \mid i+1}=J_{i} A^{-1}\left(I-\hat{J}_{i}^{T} \hat{\Lambda}_{i} \hat{J}_{i} \hat{A}^{-1}\right) \tau_{i+1}^{*}
$$

With the perfect estimation $\left(\hat{A}=A, \hat{J}_{i}=J_{i}\right)$,

$$
J_{i} A^{-1}\left(I-J_{i}^{T} \Lambda_{i} J_{i} A^{-1}\right)=0
$$

Where $\Lambda_{i}$ is i level task's inertia matrix. In this sense, the response of $i$ level task is not affected by the control torque of $i+1$ level task.

\section{B. Effect of uncertainty}

The dynamic consistency of the controller is guaranteed in a situation where modeling error does not exist. However, it is almost impossible to make the estimated model match the actual model precisely. Therefore, when controlling a real robot using the null space projector, uncertainty should be considered. In this sense, the dynamic uncertainty on the robot's mass and COM, and the kinematic uncertainty on the link length of the robot and misplacement of joints should be considered.

The inverse matrix of the robot's inertia matrix and the jacobian matrix considering modeling error are defined as follows:

$$
A^{-1}=\hat{A}^{-1}+E_{d}, \quad J_{i}=\hat{J}_{i}+E_{k_{i}}
$$

Substituting (7) with (9)

$$
\ddot{x}_{i \mid i+1}=\left(\hat{J}_{i}+E_{k_{i}}\right)\left(\hat{A}^{-1}+E_{d}\right)\left(I-\hat{J}_{i}^{T} \hat{\Lambda}_{i} \hat{J}_{i} \hat{A}^{-1}\right) \tau_{i+1}^{*}
$$

and developing (10), the response becomes

$$
\begin{array}{r}
\ddot{x}_{i \mid i+1}=\left(\hat{J}_{i} \hat{A}^{-1}+E\right)\left(I-\hat{J}_{i}^{T} \hat{\Lambda}_{i} \hat{J}_{i} \hat{A}^{-1}\right) \tau_{i+1}^{*} \\
E=E_{k_{i}} \hat{A}^{-1}+\hat{J}_{i} E_{d}+E_{k_{i}} E_{d}
\end{array}
$$

Even the tasks are decoupled with dynamic consistency of the estimated value, the error term $\left(E\left(I-\hat{J}_{i}^{T} \hat{\Lambda}_{i} \hat{J}_{i} \hat{A}^{-1}\right)\right)$ remains unless $E_{k_{i}}$ and $E_{d}$ disappear. Therefore, the coupled response of the controller is as follows:

$$
\ddot{x}_{i}=J_{i} A^{-1} \tau_{i}^{*}+E\left(I-\hat{J}_{i}^{T} \hat{\Lambda}_{i} \hat{J}_{i} \hat{A}^{-1}\right) \tau_{i+1}^{*}
$$

\section{NUMERICAL ANALYSIS AND VERIFICATION}

To evaluate (13), numerical analysis was conducted. A simulation using a planar 4-DOF manipulator and the dynamics obtained by the recursive Newton-Euler method is implemented in a matlab. Gravity and coriolis\&centrifugal forces are completely compensated to confirm the task coupling effect.

Three tasks were assigned, where only the second task was set to move along the cubic trajectory. Note that the uncertainties were considered as $+10 \%$ quantities of both dynamic and kinematic parameters

Fig. 2 shows the effect of coupling torque generated from the second task. In the case without uncertainty,the control torque of the second task does not affect the response of the first task which has high priority. It can be seen that the third task which has the lowest priority cannot follow the desired
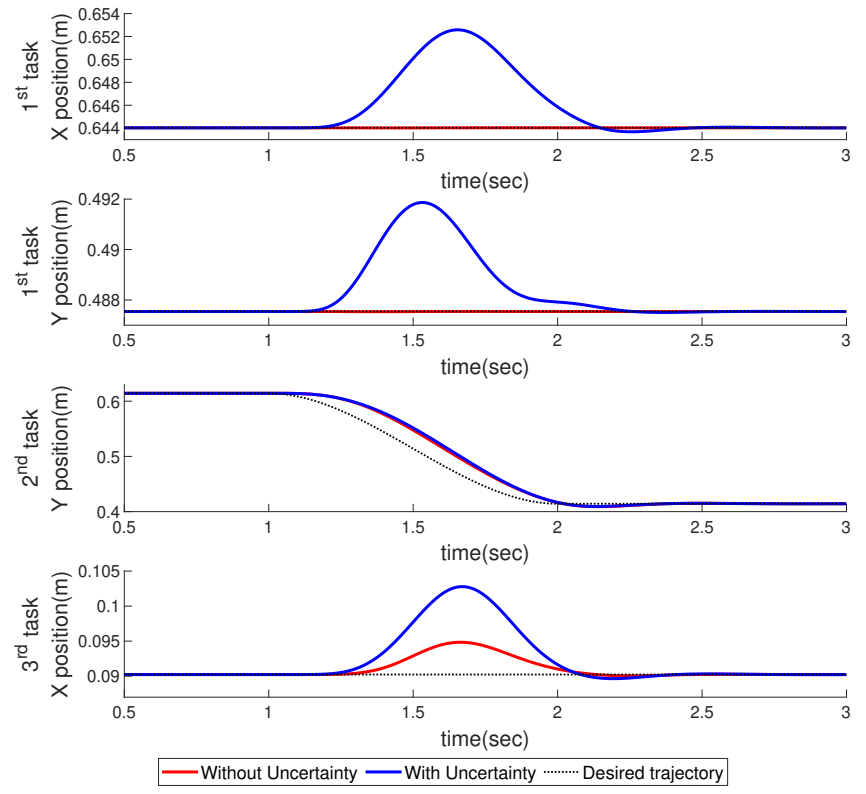

Fig. 2. Result of numerical analysis : first and second graphs show the results of the first task, i.e., the highest priority task.The third and the last plots show the results of the second and the third tasks, respectively which has lower priority.

trajectory (black line) since it is affected by control torque of the tasks that have high priority. In the case with uncertainty, it can be shown that the first task with the highest priority is affected by control torque from the tasks with lower priority due to uncertainty from modelling error.

\section{CONCLUSION}

Using the null space projector for the priority control is considered as one of a basic tools to control redundant robots. However, studies to ascertain whether the method guarantees its effectiveness are not taken seriously. This paper aims to provoke the uncertainty issue of using the null space projector as a task decoupling controller. In this sense, this paper covers the null space projector's task decoupling characteristics and the effect of uncertainty. With analytical solution and simulations, the effect of uncertainty on task coupling property of the null space projector was proven in this research.

\section{REFERENCES}

[1] O. Khatib, "A unified approach for motion and force control of robot manipulators: The operational space formulation," IEEE Journal on Robotics and Automation, vol. 3, no. 1, pp. 43-53, 1987.

[2] Y. Oh, W. Chung, and Y. Youm, "General task execution of redundant manipulators with explicit null-motion control," in Proceedings of the 1996 IEEE IECON. 22nd International Conference on Industrial Electronics, Control, and Instrumentation, vol. 3. IEEE, 1996, pp. 1902-1908.

[3] L. Sentis and O. Khatib, "A whole-body control framework for humanoids operating in human environments," in Proceedings 2006 IEEE International Conference on Robotics and Automation, 2006. ICRA 2006. IEEE, 2006, pp. 2641-2648.

[4] A. Dietrich, C. Ott, and A. Albu-Schäffer, "An overview of null space projections for redundant, torque-controlled robots," The International Journal of Robotics Research, vol. 34, no. 11, pp. 1385-1400, 2015. 